\title{
ON A TERNARY DIOPHANTINE PROBLEM WITH MIXED POWERS OF PRIMES
}

\author{
ALESSANDRO LANGUASCO \& ALESSANDRO ZACCAGNINI
}

\begin{abstract}
Let $1<k<33 / 29$. We prove that if $\lambda_{1}, \lambda_{2}$ and $\lambda_{3}$ are non-zero real numbers, not all of the same sign and that $\lambda_{1} / \lambda_{2}$ is irrational and $\varpi$ is any real number, then for any $\varepsilon>0$ the inequality $\left|\lambda_{1} p_{1}+\lambda_{2} p_{2}^{2}+\lambda_{3} p_{3}^{k}+\varpi\right| \leq\left(\max _{j} p_{j}\right)^{-(33-29 k) /(72 k)+\varepsilon}$ has infinitely many solutions in prime variables $p_{1}, \ldots, p_{k}$.
\end{abstract}

\section{INTRODUCTION}

The goal of this paper is to solve a ternary Diophantine approximation problem which involves real powers of prime numbers. We restrict our attention to the values of the form $\lambda_{1} p_{1}+\lambda_{2} p_{2}^{2}+\lambda_{3} p_{3}^{k}$, where $k \in \mathbb{R}$ and $k>1$, but similar cases can be attacked with this approach as well, see, e.g., [9]. Our main result is

Theorem 1. Let $1<k<33 / 29$ be a real number and assume that $\lambda_{1}, \lambda_{2}$ and $\lambda_{3}$ are non-zero real numbers, not all of the same sign and that $\lambda_{1} / \lambda_{2}$ is irrational. Let $\varpi$ be any real number. For any $\varepsilon>0$ the inequality

$$
\left|\lambda_{1} p_{1}+\lambda_{2} p_{2}^{2}+\lambda_{3} p_{3}^{k}+\varpi\right| \leq\left(\max _{j} p_{j}\right)^{-(33-29 k) /(72 k)+\varepsilon}
$$

has infinitely many solutions in prime variables $p_{1}, \ldots, p_{k}$.

The proof of Theorem 1 uses the variant of the circle method introduced by Davenport and Heilbronn to deal with Diophantine problems. Classical papers on this topic with integral $k$ are Vaughan's ones, [13, 14], Baker and Harman [1], Harman [5]. For nonintegral $k$ we recall that Tolev [12] studied the values of the form $p_{1}^{k}+p_{2}^{k}+p_{3}^{k}$ and proved that, for every $k \in(1,15 / 14)$, all sufficiently large real numbers $\varpi$ can be well approximated.

In order to deal with a problem with mixed non-integral powers, like the present one, a key tool is a suitable estimate for the $L^{2}$-norms of exponential sums over prime powers, see Theorems 2 and 3 of $\$ 3$, which has some independent interest. These results allow us to have a comparatively wide "major arc" while keeping the resulting error term under control. This idea appeared in Brüdern, Cook and Perelli [2] and we exploit it also in [8] and [9].

Acknowledgement. We thank Alberto Perelli for a discussion.

\section{Proof of Theorem 1}

We use the variant of the circle method introduced by Davenport and Heilbronn to deal with Diophantine problems. In order to prove that (1) has infinitely many solutions, it is sufficient to construct an increasing sequence $X_{n}$ with limit $+\infty$ such that (1) has at least a solution with $\max _{j} p_{j} \in\left[\delta X_{n}, X_{n}\right]$, where $\delta$ is a small, fixed positive constant.

2010 Mathematics Subject Classification. Primary: 11P55; secondary: 11N05.

Key words and phrases. Diophantine problems with primes, Primes in short intervals. 
This sequence actually depends on rational approximations for $\lambda_{1} / \lambda_{2}$ : more precisely, there are infinitely many pairs of integers $a$ and $q$ such that $(a, q)=1, q>0$ and

$$
\left|\frac{\lambda_{1}}{\lambda_{2}}-\frac{a}{q}\right| \leq \frac{1}{q^{2}}
$$

We take the sequence $X=q^{9 k /(2 k+3)}$ (dropping the useless suffix $n$ ) and then, as customary, define all of the circle-method parameters in terms of $X$. We may obviously assume that $q$ is sufficiently large. The choice of the exponent of $q$ and of all the other parameters is justified in the discussion in $\$ 2.7$.

Let

$$
S_{k}(\alpha)=\sum_{X \leq p^{k} \leq 2 X} \log p e\left(p^{k} \alpha\right)
$$

As usual, we approximate to $S_{k}$ using the function

$$
T_{k}(\alpha)=\int_{(\delta X)^{1 / k}}^{X^{1 / k}} e\left(t^{k} \alpha\right) \mathrm{d} t
$$

and notice the simple inequality

$$
T_{k}(\alpha) \ll_{\delta, k} X^{1 / k-1} \min \left(X,|\alpha|^{-1}\right) .
$$

We detect solutions of (1) by means of the function $\widehat{K}_{\eta}(\alpha)=\max (0, \eta-|\alpha|)$ for $\eta>0$, which, as the notation suggests, is the Fourier transform of

$$
K_{\eta}(\alpha)=\left(\frac{\sin (\pi \eta \alpha)}{\pi \alpha}\right)^{2}
$$

for $\alpha \neq 0$, and, by continuity, $K_{\eta}(0)=\eta^{2}$. This relation transforms the problem of counting solutions of the inequality (11) into estimating suitable integrals. We recall the trivial property

$$
K_{\eta}(\alpha) \ll \min \left(\eta^{2},|\alpha|^{-2}\right) .
$$

For any measurable subset $\mathfrak{X}$ of $\mathbb{R}$ let

$$
I(\eta, \varpi, \mathfrak{X})=\int_{\mathfrak{X}} S_{1}\left(\lambda_{1} \alpha\right) S_{2}\left(\lambda_{2} \alpha\right) S_{k}\left(\lambda_{3} \alpha\right) K_{\eta}(\alpha) e(\varpi \alpha) \mathrm{d} \alpha .
$$

In practice, we take as $\mathfrak{X}$ either an interval or a half line, or the union of two such sets. The starting point of the method is the observation that

$$
\begin{aligned}
I(\eta, \varpi, \mathbb{R}) & =\sum_{p_{1}, p_{2}^{2}, p_{3}^{k} \in[\delta X, X]} \log p_{1} \log p_{2} \log p_{3} \int_{\mathbb{R}} K_{\eta}(\alpha) e\left(\left(\lambda_{1} p_{1}+\lambda_{2} p_{2}^{2}+\lambda_{3} p_{3}^{k}+\varpi\right) \alpha\right) \mathrm{d} \alpha \\
& =\sum_{p_{1}, p_{2}^{2}, p_{3}^{k} \in[\delta X, X]} \log p_{1} \log p_{2} \log p_{3} \max \left(0, \eta-\left|\lambda_{1} p_{1}+\lambda_{2} p_{2}^{2}+\lambda_{3} p_{3}^{k}+\varpi\right|\right) \\
& \leq \eta(\log X)^{3} \mathcal{N}(X),
\end{aligned}
$$

where $\mathcal{N}(X)$ denotes the number of solutions of the inequality (1) with $p_{1}, p_{2}^{2}, p_{3}^{k} \in$ $[\delta X, X]$. We now give the definitions that we need to set up the method. More definitions will be given at appropriate places later. We let $P=P(X)=X^{4 /(5 k)-\varepsilon}, \eta=\eta(X)=$ $X^{-(33-29 k) /(72 k)+\varepsilon}$, and $R=R(X)=\eta^{-2} X^{(k-1) /(4 k)}(\log X)^{3}$. The choice for $P$ is justified at the end of $\$ 2.4$, the one for $\eta$ at the end of $\$ 2.5$ and the one for $R$ at the end of $\$ 2.6$. See also the discussion in $\$ 2.7$ for a more detailed argument. We now decompose $\mathbb{R}$ as $\mathfrak{M} \cup \mathfrak{m} \cup \mathfrak{t}$ where

$$
\mathfrak{M}=\left[-\frac{P}{X}, \frac{P}{X}\right], \quad \mathfrak{m}=\left(-R,-\frac{P}{X}\right) \cup\left(\frac{P}{X}, R\right), \quad \mathfrak{t}=\mathbb{R} \backslash(\mathfrak{M} \cup \mathfrak{m}),
$$


so that

$$
I(\eta, \varpi, \mathbb{R})=I(\eta, \varpi, \mathfrak{M})+I(\eta, \varpi, \mathfrak{m})+I(\eta, \varpi, \mathfrak{t}) .
$$

These sets are called the major arc, the intermediate (or minor) arc and the trivial arc respectively. In $\$ 2.1$ we prove that the major arc yields the main term for $I(\eta, \varpi, \mathbb{R})$. In order to show that the contribution of the intermediate arc does not cancel the main term, we exploit the hypothesis that $\lambda_{1} / \lambda_{2}$ is irrational to prove that $\left|S_{1}\left(\lambda_{1} \alpha\right)\right|^{1 / 2}$ and $\left|S_{2}\left(\lambda_{2} \alpha\right)\right|$ can not both be large for $\alpha \in \mathfrak{m}$ : see $\$ 2.5$, and in particular Lemma 3, for the details. The trivial arc, treated in $\$ 2.6$, only gives a rather small contribution.

From now on, implicit constants may depend on the coefficients $\lambda_{j}$, on $k, \delta$ and $\varpi$.

2.1. The major arc. We write

$$
\begin{aligned}
I(\eta, \varpi, \mathfrak{M})= & \int_{\mathfrak{M}} S_{1}\left(\lambda_{1} \alpha\right) S_{2}\left(\lambda_{2} \alpha\right) S_{k}\left(\lambda_{3} \alpha\right) K_{\eta}(\alpha) e(\varpi \alpha) \mathrm{d} \alpha \\
= & \int_{\mathfrak{M}} T_{1}\left(\lambda_{1} \alpha\right) T_{2}\left(\lambda_{2} \alpha\right) T_{k}\left(\lambda_{3} \alpha\right) K_{\eta}(\alpha) e(\varpi \alpha) \mathrm{d} \alpha \\
& \quad+\int_{\mathfrak{M}}\left(S_{1}\left(\lambda_{1} \alpha\right)-T_{1}\left(\lambda_{1} \alpha\right)\right) T_{2}\left(\lambda_{2} \alpha\right) T_{k}\left(\lambda_{3} \alpha\right) K_{\eta}(\alpha) e(\varpi \alpha) \mathrm{d} \alpha \\
& \quad+\int_{\mathfrak{M}} S_{1}\left(\lambda_{1} \alpha\right)\left(S_{2}\left(\lambda_{2} \alpha\right)-T_{2}\left(\lambda_{2} \alpha\right)\right) T_{k}\left(\lambda_{3} \alpha\right) K_{\eta}(\alpha) e(\varpi \alpha) \mathrm{d} \alpha \\
& \quad+\int_{\mathfrak{M}} S_{1}\left(\lambda_{1} \alpha\right) S_{2}\left(\lambda_{2} \alpha\right)\left(S_{k}\left(\lambda_{3} \alpha\right)-T_{k}\left(\lambda_{3} \alpha\right)\right) K_{\eta}(\alpha) e(\varpi \alpha) \mathrm{d} \alpha \\
= & J_{1}+J_{2}+J_{3}+J_{4},
\end{aligned}
$$

say. We will give a lower bound for $J_{1}$ and upper bounds for $J_{2}, \ldots, J_{4}$. For brevity, since the computations for $J_{3}$ are similar to, but simpler than, the corresponding ones for $J_{2}$ and $J_{4}$, we will skip them.

2.2. Lower bound for $J_{1}$. The lower bound $J_{1} \gg \eta^{2} X^{1 / 2+1 / k}$ is proved in a classical way. We have

$$
\begin{aligned}
J_{1}= & \int_{\mathfrak{M}} T_{1}\left(\lambda_{1} \alpha\right) T_{2}\left(\lambda_{2} \alpha\right) T_{k}\left(\lambda_{3} \alpha\right) K_{\eta}(\alpha) e(\varpi \alpha) \mathrm{d} \alpha \\
= & \int_{\mathbb{R}} T_{1}\left(\lambda_{1} \alpha\right) T_{2}\left(\lambda_{2} \alpha\right) T_{k}\left(\lambda_{3} \alpha\right) K_{\eta}(\alpha) e(\varpi \alpha) \mathrm{d} \alpha \\
& \quad+\mathcal{O}\left(\int_{P / X}^{+\infty}\left|T_{1}\left(\lambda_{1} \alpha\right) T_{2}\left(\lambda_{2} \alpha\right) T_{k}\left(\lambda_{3} \alpha\right)\right| K_{\eta}(\alpha) \mathrm{d} \alpha\right) .
\end{aligned}
$$

Using inequalities (3) and (44), we see that the error term is

$$
\ll \eta^{2} X^{1 / k-3 / 2} \int_{P / X}^{+\infty} \frac{\mathrm{d} \alpha}{\alpha^{3}} \ll \eta^{2} X^{1 / 2+1 / k} P^{-2}=o\left(\eta^{2} X^{1 / 2+1 / k}\right) .
$$

For brevity, we set $\mathfrak{D}=[\delta X, X] \times\left[(\delta X)^{1 / 2}, X^{1 / 2}\right] \times\left[(\delta X)^{1 / k}, X^{1 / k}\right]$. We can rewrite the main term in the form

$$
\begin{aligned}
& \int \cdots \int_{\mathfrak{D}} \int_{\mathbb{R}} e\left(\left(\lambda_{1} t_{1}+\lambda_{2} t_{2}^{2}+\lambda_{3} t_{3}^{k}+\varpi\right) \alpha\right) K_{\eta}(\alpha) \mathrm{d} \alpha \mathrm{d} t_{1} \mathrm{~d} t_{2} \mathrm{~d} t_{3} \\
& =\int \cdots \int_{\mathfrak{D}} \max \left(0, \eta-\left|\lambda_{1} t_{1}+\lambda_{2} t_{2}^{2}+\lambda_{3} t_{3}^{k}+\varpi\right|\right) \mathrm{d} t_{1} \mathrm{~d} t_{2} \mathrm{~d} t_{3} .
\end{aligned}
$$

We now proceed to show that the last integral is $\gg \eta^{2} X^{1 / 2+1 / k}$. Apart from trivial changes of sign, there are essentially three cases: 
(1) $\lambda_{1}>0, \lambda_{2}<0, \lambda_{3}<0$

(2) $\lambda_{1}>0, \lambda_{2}>0, \lambda_{3}<0$

(3) $\lambda_{1}>0, \lambda_{2}<0, \lambda_{3}>0$.

We briefly deal with the second case, the other ones being similar. A suitable change of variables shows that

$$
\begin{aligned}
J_{1} & \gg \int \cdots \int_{\mathfrak{D}^{\prime}} \max \left(0, \eta-\left|\lambda_{1} u_{1}+\lambda_{2} u_{2}+\lambda_{3} u_{3}\right|\right) \frac{\mathrm{d} u_{1} \mathrm{~d} u_{2} \mathrm{~d} u_{3}}{u_{2}^{1 / 2} u_{3}^{1-1 / k}} \\
& \gg X^{1 / k-3 / 2} \int \cdots \int_{\mathfrak{D}^{\prime}} \max \left(0, \eta-\left|\lambda_{1} u_{1}+\lambda_{2} u_{2}+\lambda_{3} u_{3}\right|\right) \mathrm{d} u_{1} \mathrm{~d} u_{2} \mathrm{~d} u_{3},
\end{aligned}
$$

where $\mathfrak{D}^{\prime}=[\delta X,(1-\delta) X]^{3}$, for large $X$. For $j=1,2$, let $a_{j}=\left|\lambda_{3}\right| \delta /\left|\lambda_{j}\right|, b_{j}=2 a_{j}$ and $\mathfrak{I}_{j}=\left[a_{j} X, b_{j} X\right]$. Notice that if $u_{j} \in \mathfrak{I}_{j}$ for $j=1,2$, then

$$
\lambda_{1} u_{1}+\lambda_{2} u_{2} \in\left[2\left|\lambda_{3}\right| \delta X, 4\left|\lambda_{3}\right| \delta X\right]
$$

so that, for every such choice of $\left(u_{1}, u_{2}\right)$, the interval $[a, b]$ with endpoints $\pm \eta /\left|\lambda_{3}\right|+$ $\left(\lambda_{1} u_{1}+\lambda_{2} u_{2}\right) /\left|\lambda_{3}\right|$ is contained in $[\delta X,(1-\delta) X]$. In other words, for $u_{3} \in[a, b]$ the values of $\lambda_{1} u_{1}+\lambda_{2} u_{2}+\lambda_{3} u_{3}$ cover the whole interval $[-\eta, \eta]$. Hence, for any $\left(u_{1}, u_{2}\right) \in \mathfrak{I}_{1} \times \mathfrak{I}_{2}$, we have

$$
\int_{\delta X}^{(1-\delta) X} \max \left(0, \eta-\left|\lambda_{1} u_{1}+\lambda_{2} u_{2}+\lambda_{3} u_{3}\right|\right) \mathrm{d} u_{3}=\frac{1}{\left|\lambda_{3}\right|} \int_{-\eta}^{\eta} \max (0, \eta-|u|) \mathrm{d} u \gg \eta^{2} .
$$

Finally,

$$
J_{1} \gg \eta^{2} X^{1 / k-3 / 2} \iint_{\mathfrak{I}_{1} \times \mathfrak{I}_{2}} \mathrm{~d} u_{1} \mathrm{~d} u_{2} \gg \eta^{2} X^{1 / 2+1 / k},
$$

which is the required lower bound.

2.3. Bound for $J_{2}$. We define another approximation of $S_{k}(\alpha)$, namely

$$
U_{k}(\alpha)=\sum_{X \leq n^{k} \leq 2 X} e\left(n^{k} \alpha\right)
$$

The Euler summation formula implies that

$$
T_{k}(\alpha)-U_{k}(\alpha) \ll 1+|\alpha| X .
$$

Using (4) we see that

$$
\begin{aligned}
J_{2} & \ll \eta^{2} \int_{\mathfrak{M}}\left|S_{1}\left(\lambda_{1} \alpha\right)-T_{1}\left(\lambda_{1} \alpha\right)\right|\left|T_{2}\left(\lambda_{2} \alpha\right)\right|\left|T_{k}\left(\lambda_{3} \alpha\right)\right| \mathrm{d} \alpha \\
\leq & \eta^{2} \int_{\mathfrak{M}}\left|S_{1}\left(\lambda_{1} \alpha\right)-U_{1}\left(\lambda_{1} \alpha\right)\right|\left|T_{2}\left(\lambda_{2} \alpha\right)\right|\left|T_{k}\left(\lambda_{3} \alpha\right)\right| \mathrm{d} \alpha \\
& \quad+\eta^{2} \int_{\mathfrak{M}}\left|U_{1}\left(\lambda_{1} \alpha\right)-T_{1}\left(\lambda_{1} \alpha\right)\right|\left|T_{2}\left(\lambda_{2} \alpha\right)\right|\left|T_{k}\left(\lambda_{3} \alpha\right)\right| \mathrm{d} \alpha \\
& =\eta^{2}\left(A_{2}+B_{2}\right),
\end{aligned}
$$

say. In order to estimate $A_{2}$ we use Theorems 2 and 3 . By the Cauchy inequality and (3) above, for any fixed $A>0$ we have

$$
\begin{aligned}
A_{2} & \ll\left(\int_{-P / X}^{P / X}\left|S_{1}\left(\lambda_{1} \alpha\right)-U_{1}\left(\lambda_{1} \alpha\right)\right|^{2} \mathrm{~d} \alpha\right)^{1 / 2}\left(\int_{-P / X}^{P / X}\left|T_{2}\left(\lambda_{2} \alpha\right)\right|^{2}\left|T_{k}\left(\lambda_{3} \alpha\right)\right|^{2} \mathrm{~d} \alpha\right)^{1 / 2} \\
& \ll\left(\frac{X}{(\log X)^{A}}\right)^{1 / 2}\left(\int_{0}^{1 / X} X^{1+2 / k} \mathrm{~d} \alpha+\int_{1 / X}^{P / X} \frac{X^{2 / k-3}}{\alpha^{4}} \mathrm{~d} \alpha\right)^{1 / 2}
\end{aligned}
$$




$$
\ll_{A} \frac{X^{1 / 2+1 / k}}{(\log X)^{A / 2}}
$$

by Theorem 3 (with $C=12 / 5$ ), which we can use provided that $X / P \geq X^{1 / 6+\varepsilon}$, that is, $P \leq X^{5 / 6-\varepsilon}$. This proves that $\eta^{2} A_{2}=o\left(\eta^{2} X^{1 / 2+1 / k}\right)$. Furthermore, using the inequalities (3) and (6) we see that

$$
\begin{aligned}
B_{2} & \ll \int_{0}^{1 / X}\left|T_{2}\left(\lambda_{2} \alpha\right)\right|\left|T_{k}\left(\lambda_{3} \alpha\right)\right| \mathrm{d} \alpha+X \int_{1 / X}^{P / X} \alpha\left|T_{2}\left(\lambda_{2} \alpha\right)\right|\left|T_{k}\left(\lambda_{3} \alpha\right)\right| \mathrm{d} \alpha \\
& \ll X^{1 / k-1 / 2}+X^{1 / k-1 / 2} \int_{1 / X}^{P / X} \frac{\mathrm{d} \alpha}{\alpha} \ll X^{1 / k-1 / 2} \log P
\end{aligned}
$$

so that $\eta^{2} B_{2}=o\left(\eta^{2} X^{1 / 2+1 / k}\right)$.

2.4. Bound for $J_{4}$. Inequality (4) implies that

$$
\begin{aligned}
J_{4} & \ll \eta^{2} \int_{\mathfrak{M}}\left|S_{1}\left(\lambda_{1} \alpha\right)\right|\left|S_{2}\left(\lambda_{2} \alpha\right)\right|\left|S_{k}\left(\lambda_{3} \alpha\right)-T_{k}\left(\lambda_{3} \alpha\right)\right| \mathrm{d} \alpha \\
\ll & \ll \eta^{2} \int_{\mathfrak{M}}\left|S_{1}\left(\lambda_{1} \alpha\right)\right|\left|S_{2}\left(\lambda_{2} \alpha\right)\right|\left|S_{k}\left(\lambda_{3} \alpha\right)-U_{k}\left(\lambda_{3} \alpha\right)\right| \mathrm{d} \alpha \\
& \quad+\eta^{2} \int_{\mathfrak{M}}\left|S_{1}\left(\lambda_{1} \alpha\right)\right|\left|S_{2}\left(\lambda_{2} \alpha\right)\right|\left|U_{k}\left(\lambda_{3} \alpha\right)-T_{k}\left(\lambda_{3} \alpha\right)\right| \mathrm{d} \alpha \\
& =\eta^{2}\left(A_{4}+B_{4}\right),
\end{aligned}
$$

say. The Parseval inequality and trivial bounds yield, for any fixed $A>0$,

$$
\begin{aligned}
A_{4} & \ll X^{1 / 2}\left(\int_{\mathfrak{M}}\left|S_{1}\left(\lambda_{1} \alpha\right)\right|^{2} \mathrm{~d} \alpha\right)^{1 / 2}\left(\int_{\mathfrak{M}}\left|S_{k}\left(\lambda_{3} \alpha\right)-U_{k}\left(\lambda_{3} \alpha\right)\right|^{2} \mathrm{~d} \alpha\right)^{1 / 2} \\
& \ll X(\log X)^{1 / 2} \frac{P}{X} J_{k}\left(X, \frac{X}{P}\right)^{1 / 2} \ll_{A} X^{1 / 2+1 / k}(\log X)^{1 / 2-A / 2}
\end{aligned}
$$

by Theorems 2 and 3 (with $C=12 / 5$ ) which we can use provided that $X / P \geq X^{1-5 /(6 k)+\varepsilon}$, that is, $P \leq X^{5 /(6 k)-\varepsilon}$. This proves that $\eta^{2} A_{4}=o\left(\eta^{2} X^{1 / 2+1 / k}\right)$. Furthermore, using (6), the Hölder inequality and trivial bounds we see that

$$
\begin{aligned}
B_{4} & \ll \int_{0}^{1 / X}\left|S_{1}\left(\lambda_{1} \alpha\right)\right|\left|S_{2}\left(\lambda_{2} \alpha\right)\right| \mathrm{d} \alpha+X \int_{1 / X}^{P / X} \alpha\left|S_{1}\left(\lambda_{1} \alpha\right)\right|\left|S_{2}\left(\lambda_{2} \alpha\right)\right| \mathrm{d} \alpha \\
& \ll X^{1 / 2}+X\left(\int_{1 / X}^{P / X}\left|S_{1}\left(\lambda_{1} \alpha\right)\right|^{2} \mathrm{~d} \alpha\right)^{1 / 2}\left(\int_{1 / X}^{P / X}\left|S_{2}\left(\lambda_{2} \alpha\right)\right|^{4} \mathrm{~d} \alpha \int_{1 / X}^{P / X} \alpha^{4} \mathrm{~d} \alpha\right)^{1 / 4} \\
& \ll X(X \log X)^{1 / 2}\left(X(\log X)^{2}\right)^{1 / 4}\left(\frac{P}{X}\right)^{5 / 4} \ll P^{5 / 4} X^{1 / 2} \log X .
\end{aligned}
$$

Here we used Satz 3 of Rieger [10] to bound the fourth moment of $S_{2}$. Hence, taking $P=o\left(X^{4 /(5 k)}(\log X)^{-1}\right)$ we get $\eta^{2} B_{4}=o\left(\eta^{2} X^{1 / 2+1 / k}\right)$. We may therefore choose

$$
P=X^{4 /(5 k)-\varepsilon} \text {. }
$$

2.5. The intermediate arc. We need to show that $\left|S_{1}\left(\lambda_{1} \alpha\right)\right|^{1 / 2}$ and $\left|S_{2}\left(\lambda_{2} \alpha\right)\right|$ can not both be large for $\alpha \in \mathfrak{m}$, exploiting the fact that $\lambda_{1} / \lambda_{2}$ is irrational. We do this using two famous results by Vaughan and Ghosh, respectively, about $S_{1}(\alpha)$ and $S_{2}(\alpha)$. 
Lemma 1 (Vaughan [15], Theorem 3.1). Let $\alpha$ be a real number and $a, q$ be positive integers satisfying $(a, q)=1$ and $|\alpha-a / q|<q^{-2}$. Then

$$
S_{1}(\alpha) \ll\left(\frac{X}{\sqrt{q}}+\sqrt{X q}+X^{4 / 5}\right) \log ^{4} X .
$$

Lemma 2 (Ghosh [4], Theorem 2). Let $\alpha$ be a real number and a, $q$ be positive integers satisfying $(a, q)=1$ and $|\alpha-a / q|<q^{-2}$. Let moreover $\varepsilon>0$. Then

$$
S_{2}(\alpha) \ll_{\varepsilon} X^{1 / 2+\varepsilon}\left(\frac{1}{q}+\frac{1}{X^{1 / 4}}+\frac{q}{X}\right)^{1 / 4} .
$$

Lemma 3. Let $1 \leq k<33 / 29$. Assume that $\lambda_{1} / \lambda_{2}$ is irrational and let $X=q^{9 k /(2 k+3)}$, where $q$ is the denominator of a convergent of the continued fraction for $\lambda_{1} / \lambda_{2}$. Let $V(\alpha)=\min \left(\left|S_{1}\left(\lambda_{1} \alpha\right)\right|^{1 / 2},\left|S_{2}\left(\lambda_{2} \alpha\right)\right|\right)$. Then we have

$$
\sup _{\alpha \in \mathfrak{m}} V(\alpha) \ll X^{(29 k+3) /(72 k)+\varepsilon} .
$$

Proof. Let $\alpha \in \mathfrak{m}$ and $Q=X^{(7 k-3) /(18 k)} \leq P$. By Dirichlet's Theorem, there exist integers $a_{i}, q_{i}$ with $1 \leq q_{i} \leq X / Q$ and $\left(a_{i}, q_{i}\right)=1$, such that $\left|\lambda_{i} \alpha q_{i}-a_{i}\right| \leq Q / X$, for $i=1,2$. We remark that $a_{1} a_{2} \neq 0$, for otherwise we would have $\alpha \in \mathfrak{M}$. Now suppose that $q_{i} \leq Q$ for $i=1,2$. In this case we get

and hence

$$
a_{2} q_{1} \frac{\lambda_{1}}{\lambda_{2}}-a_{1} q_{2}=\left(\lambda_{1} \alpha q_{1}-a_{1}\right) \frac{a_{2}}{\lambda_{2} \alpha}-\left(\lambda_{2} \alpha q_{2}-a_{2}\right) \frac{a_{1}}{\lambda_{2} \alpha}
$$

$$
\left|a_{2} q_{1} \frac{\lambda_{1}}{\lambda_{2}}-a_{1} q_{2}\right| \leq 2\left(1+\left|\frac{\lambda_{1}}{\lambda_{2}}\right|\right) \frac{Q^{2}}{X}<\frac{1}{2 q}
$$

for sufficiently large $X$. Then, from the law of best approximation and the definition of $\mathfrak{m}$, we obtain

$$
X^{(2 k+3) /(9 k)}=q \leq\left|a_{2} q_{1}\right| \ll q_{1} q_{2} R \leq Q^{2} R \leq X^{(2 k+3) /(9 k)-\varepsilon},
$$

which is absurd. Hence either $q_{1}>Q$ or $q_{2}>Q$. Assume that $q_{1}>Q$. Using Lemma 1 on $S_{1}\left(\lambda_{1} \alpha\right)$, we have

$$
\begin{aligned}
& V(\alpha) \leq\left|S_{1}\left(\lambda_{1} \alpha\right)\right|^{1 / 2} \ll \sup _{Q<q_{1} \leq X / Q}\left(\frac{X}{\sqrt{q_{1}}}+\sqrt{X q_{1}}+X^{4 / 5}\right)^{1 / 2} \log ^{2} X \\
& \ll X^{(29 k+3) /(72 k)}(\log X)^{2} .
\end{aligned}
$$

The other case is similar, using Lemma 2 instead, and hence Lemma 3 follows.

Lemma 4. For $j=1,2$ we have

$$
\begin{aligned}
& \int_{\mathfrak{m}}\left|S_{j}\left(\lambda_{j} \alpha\right)\right|^{2 j} K_{\eta}(\alpha) \mathrm{d} \alpha \ll \eta X(\log X)^{j} \\
& \int_{\mathfrak{m}}\left|S_{k}\left(\lambda_{3} \alpha\right)\right|^{2} K_{\eta}(\alpha) \mathrm{d} \alpha \ll \eta X^{1 / k}(\log X)^{3} .
\end{aligned}
$$

Proof. The proof is achieved arguing as in $\$ 2.6$ below where we bound the quantities $A$, $B$ and $C$, the main difference being the fact that we have to split the range $[P / X, R]$ into two intervals in order to use (4) efficiently. See also the proof of Lemma 7 of Tolev [12. For the sake of brevity we skip the details.

Now let

$$
\mathfrak{X}_{1}=\left\{\alpha \in[P / X, R]:\left|S_{1}\left(\lambda_{1} \alpha\right)\right|^{1 / 2} \leq\left|S_{2}\left(\lambda_{2} \alpha\right)\right|\right\}
$$




$$
\mathfrak{X}_{2}=\left\{\alpha \in[P / X, R]:\left|S_{1}\left(\lambda_{1} \alpha\right)\right|^{1 / 2} \geq\left|S_{2}\left(\lambda_{2} \alpha\right)\right|\right\}
$$

so that $[P / X, R]=\mathfrak{X}_{1} \cup \mathfrak{X}_{2}$ and

$$
|I(\eta, \varpi, \mathfrak{m})| \ll\left(\int_{\mathfrak{X}_{1}}+\int_{\mathfrak{X}_{2}}\right)\left|S_{1}\left(\lambda_{1} \alpha\right) S_{2}\left(\lambda_{2} \alpha\right) S_{k}\left(\lambda_{3} \alpha\right)\right| K_{\eta}(\alpha) \mathrm{d} \alpha .
$$

Hölder's inequality gives

$$
\begin{aligned}
\int_{\mathfrak{X}_{1}} \leq & \max _{\alpha \in \mathfrak{X}_{1}}\left|S_{1}\left(\lambda_{1} \alpha\right)\right|^{1 / 2}\left(\int_{\mathfrak{X}_{1}}\left|S_{1}\left(\lambda_{1} \alpha\right)\right|^{2} K_{\eta}(\alpha) \mathrm{d} \alpha\right)^{1 / 4} \times \\
& \left(\int_{\mathfrak{X}_{1}}\left|S_{2}\left(\lambda_{2} \alpha\right)\right|^{4} K_{\eta}(\alpha) \mathrm{d} \alpha\right)^{1 / 4}\left(\int_{\mathfrak{X}_{1}}\left|S_{k}\left(\lambda_{3} \alpha\right)\right|^{2} K_{\eta}(\alpha) \mathrm{d} \alpha\right)^{1 / 2} \\
& \ll \eta X^{(65 k+39) /(72 k)+\varepsilon}
\end{aligned}
$$

by Lemmas 3 and 4 . The computation on $\mathfrak{X}_{2}$ is similar and gives the same final result. Summing up,

$$
|I(\eta, \varpi, \mathfrak{m})| \ll \eta X^{(65 k+39) /(72 k)+\varepsilon}
$$

and this is $o\left(\eta^{2} X^{1 / 2+1 / k}\right)$ provided that

$$
\eta=\infty\left(X^{(29 k-33) /(72 k)+\varepsilon}\right) .
$$

2.6. The trivial arc. Using the Hölder inequality and a trivial bound for $S_{k}\left(\lambda_{3} \alpha\right)$ we see that

$$
\begin{aligned}
|I(\eta, \varpi, \mathfrak{t})| \leq & 2 \int_{R}^{+\infty}\left|S_{1}\left(\lambda_{1} \alpha\right)\right|\left|S_{2}\left(\lambda_{2} \alpha\right)\right|\left|S_{k}\left(\lambda_{3} \alpha\right)\right| K_{\eta}(\alpha) \mathrm{d} \alpha \\
\ll & \left(\int_{R}^{+\infty}\left|S_{1}\left(\lambda_{1} \alpha\right)\right|^{2} K_{\eta}(\alpha) \mathrm{d} \alpha\right)^{1 / 2}\left(\int_{R}^{+\infty}\left|S_{2}\left(\lambda_{2} \alpha\right)\right|^{4} K_{\eta}(\alpha) \mathrm{d} \alpha\right)^{1 / 4} \times \\
& \left(\int_{R}^{+\infty}\left|S_{k}\left(\lambda_{3} \alpha\right)\right|^{4} K_{\eta}(\alpha) \mathrm{d} \alpha\right)^{1 / 4} \\
\leq & X^{1 / 2 k}\left(\int_{R}^{+\infty}\left|S_{1}\left(\lambda_{1} \alpha\right)\right|^{2} K_{\eta}(\alpha) \mathrm{d} \alpha\right)^{1 / 2}\left(\int_{R}^{+\infty}\left|S_{2}\left(\lambda_{2} \alpha\right)\right|^{4} K_{\eta}(\alpha) \mathrm{d} \alpha\right)^{1 / 4} \times \\
& \left(\int_{R}^{+\infty}\left|S_{k}\left(\lambda_{3} \alpha\right)\right|^{2} K_{\eta}(\alpha) \mathrm{d} \alpha\right)^{1 / 4} \\
\ll & X^{1 / 2 k} A^{1 / 2} B^{1 / 4} C^{1 / 4},
\end{aligned}
$$

say, where in the last but one line we used the inequality (4), and we set

$$
A=\int_{\left|\lambda_{1}\right| R}^{+\infty} \frac{\left|S_{1}(\alpha)\right|^{2}}{\alpha^{2}} \mathrm{~d} \alpha, \quad B=\int_{\left|\lambda_{2}\right| R}^{+\infty} \frac{\left|S_{2}(\alpha)\right|^{4}}{\alpha^{2}} \mathrm{~d} \alpha, \quad C=\int_{\left|\lambda_{3}\right| R}^{+\infty} \frac{\left|S_{k}(\alpha)\right|^{2}}{\alpha^{2}} \mathrm{~d} \alpha .
$$

We have

$$
A \ll \sum_{n \geq\left|\lambda_{1}\right| R} \frac{1}{(n-1)^{2}} \int_{n-1}^{n}\left|S_{1}(\alpha)\right|^{2} \mathrm{~d} \alpha \ll \frac{X \log X}{\left|\lambda_{1}\right| R}
$$

by the Prime Number Theorem (PNT). Arguing similarly, using again Satz 3 of Rieger [10] and Lemma 7 of Tolev [12] respectively, we see that we also have $B \ll X(\log X)^{2} / R$ and $C \ll X^{1 / k}(\log X)^{3} / R$. Collecting these estimates, we conclude that

$$
|I(\eta, \varpi, \mathfrak{t})| \ll \frac{X^{3 / 4+3 /(4 k)}(\log X)^{2}}{R} .
$$


Hence, $|I(\eta, \varpi, \mathfrak{t})|=o\left(\eta^{2} X^{1 / 2+1 / k}\right)$ provided that we choose, say,

$$
R=\eta^{-2} X^{(1-1 / k) / 4}(\log X)^{3} .
$$

2.7. Remark on the choice of the parameters. The constraint on the choice $X=$ $q^{9 k /(2 k+3)}$ with $1<k<33 / 29$ arises from the bounds (8) and (9). Their combination prevents us from choosing the optimal value $X=q^{2}$. This is justified as follows: neglecting $\log$-powers, let $X=q^{a(k)}, Q=X^{b(k)}, \eta=X^{-c(k)}$, and recall the choices $P=X^{4 /(5 k)-\varepsilon}$ in (17) and $R=\eta^{-2} X^{(1-1 / k) / 4}(\log X)^{3}$ in (11) which are due, respectively, to the bound for $B_{4}$ and for the trivial arc. Then, essentially, we have to maximize $k$ subject to the constraints

$$
\begin{cases}a(k) \geq 1 & \\ 0 \leq b(k) \leq \frac{4}{5 k} & \\ c(k) \geq 0 & \text { by (18), } \\ 2 b(k)-1 \leq-1 / a(k) & \text { by (19), } \\ 2 b(k)+2 c(k)+\frac{1}{4}\left(1-\frac{1}{k}\right) \leq 1 / a(k) & \text { by (10), } \\ -c(k) \geq \frac{1}{2}-\frac{1}{2 k}-\frac{1}{4} b(k) & \text { (10) }\end{cases}
$$

which is a linear optimization problem in the variables $1 / a(k), b(k), c(k)$ and $1 / k$. The solution for this problem is $1 / a(k)=(2 k+3) /(9 k), b(k)=(7 k-3) /(18 k), c(k)=$ $(33-29 k) /(72 k)$, for $1 / k \geq 29 / 33$, and this is equivalent to the statement of Theorem 1 .

\section{3. $L^{2}$-NORMS OF EXPONENTIAL SUMS OVER PRIME POWERS}

In the proof of Theorem 1 we needed a mean-square average of $S_{k}(\alpha)-U_{k}(\alpha)$, respectively defined in (2) and (5), for $k>1$. In this section we see the slightly more general case $k>0$.

We need to recall that $\theta(x)=\sum_{p \leq x} \log p$ and to define the quantity

$$
J_{k}(X, h)=\int_{X}^{2 X}\left(\theta\left((x+h)^{1 / k}\right)-\theta\left(x^{1 / k}\right)-\left((x+h)^{1 / k}-x^{1 / k}\right)\right)^{2} \mathrm{~d} x
$$

which is a generalization of the Selberg integral which is well suited for our problem. To be consistent with the classical definition, we will also denote $J_{1}$ as $J$.

We want first to relate a truncated $L^{2}$-average of $S_{k}(\alpha)-U_{k}(\alpha)$ with $J_{k}(X, h)$ and then to obtain a suitable estimate for the latter.

Theorem 2. Let $k>0$ be a real number. For $0<Y \leq 1 / 2$ we have

$$
\int_{-Y}^{Y}\left|S_{k}(\alpha)-U_{k}(\alpha)\right|^{2} \mathrm{~d} \alpha \ll_{k} \frac{X^{2 / k-2} \log ^{2} X}{Y}+Y^{2} X+Y^{2} J_{k}\left(X, \frac{1}{2 Y}\right),
$$

where $J_{k}(X, h)$ is defined in (12) .

A similar result holds replacing $\log p$ with the von Mangoldt function $\Lambda(n)$ in the definition of $S_{k}(\alpha)$ in (2); the only difference in the statement above will be replacing $J_{k}$ with $J_{k, \psi}$ as defined in (17). The case $k=1$ is well known, see, e.g., Lemma 1 of Brüdern-Cook-Perelli [2].

In order to state the following result, we introduce an hypothesis on the density of the zeros of the Riemann zeta-function. With classical notation, we assume that there exist constants $B \geq 0$ and $C \geq 2$ such that for $\sigma \in[1 / 2,1]$ and $T \geq 2$ we have

$$
N(\sigma, T) \ll T^{C(1-\sigma)}(\log T)^{B} .
$$

Huxley [6] proved that (13) holds with $C=12 / 5$ and some $B \geq 0$. 
Theorem 3. Let $k>0$ be a real number and $\varepsilon$ be an arbitrarily small positive constant. Assuming that (13) holds, there exists a positive constant $c_{1}=c_{1}(\varepsilon)$, which does not depend on $k$, such that

$$
J_{k}(X, h) \ll_{k} h^{2} X^{2 / k-1} \exp \left(-c_{1}\left(\frac{\log X}{\log \log X}\right)^{1 / 3}\right)
$$

uniformly for $X^{1-2 /(C k)+\varepsilon} \leq h \leq X$. Assuming further that the Riemann Hypothesis holds, we have

uniformly for $X^{1-1 / k} \leq h \leq X$.

$$
J_{k}(X, h) \ll_{k} h X^{1 / k} \log ^{2}\left(\frac{2 X}{h}\right)
$$

Notice that if $k \geq 1$ and $h \leq X^{1-1 / k}$, then the bound $J_{k}(X, h) \ll X \log X$ follows immediately from the Prime Number Theorem. For $k<1$ the previous condition on $h$ become essentially meaningless. The case $k=1$ of the previous theorem was proved by Saffari-Vaughan [11], see $\S 6$ there, while in Zaccagnini [16] a wider range for $h$ in the unconditional case is given, but the proof does not easily lend itself to the generalization we pursue here. The unconditional case $k=2$ of Theorems 2 and 3 (with $C=12 / 5$ ) was proved in Languasco-Settimi [7]. Applications of this case to some diophantine problems with primes and squares of primes were given in [7] and in Languasco-Zaccagnini [8]. Results similar to Theorem 3 hold also replacing $\theta(x)$ with $\psi(x)$, see Lemma 5 below, and replacing $h$ with $\delta x$, see Lemma 6 .

3.1. Proof of Theorem 2, Letting $\mathcal{I}:=\int_{-Y}^{Y}\left|S_{k}(\alpha)-U_{k}(\alpha)\right|^{2} \mathrm{~d} \alpha$, we see that the result is trivial for $0<Y<1 / X$ since $\mathcal{I} \ll Y X^{2 / k} \leq Y^{-1} X^{2 / k-2} \log ^{2} X$ in this range. Assuming that $1 / X \leq Y \leq 1 / 2$, we can write

$$
\mathcal{I}=\int_{-Y}^{Y}\left|\sum_{X \leq n^{k} \leq 2 X}(\ell(n)-1) e\left(n^{k} \alpha\right)\right|^{2} \mathrm{~d} \alpha
$$

where $\ell(n)=\log p$ if $n=p$ prime and $\ell(n)=0$ otherwise. By Gallagher's lemma (Lemma 1 of [3]) we obtain

$$
\mathcal{I} \ll Y^{2} \int_{-\infty}^{\infty}\left(\sum_{\substack{x \leq n^{k} \leq x+H \\ X \leq n^{k} \leq 2 X}}(\ell(n)-1)\right)^{2} \mathrm{~d} x
$$

where we defined $H=1 /(2 Y)$. We can restrict the integration range to $E=[X-H, 2 X]$ since otherwise the inner sum is empty. Moreover we split $E$ as $E=E_{1} \cup E_{2} \cup E_{3}$ where $E_{1}=[X-H, X], E_{2}=[X, 2 X-H], E_{3}=[2 X-H, 2 X]$. Accordingly we can write

$$
\mathcal{I} \ll Y^{2}\left(\int_{E_{1}}+\int_{E_{2}}+\int_{E_{3}}\right)\left(\sum_{\substack{x \leq n^{k} \leq x+H \\ X \leq n^{k} \leq 2 X}}(\ell(n)-1)\right)^{2} \mathrm{~d} x=Y^{2}\left(I_{1}+I_{2}+I_{3}\right),
$$

say. We now proceed to estimate $I_{i}$, for every $i=1,2,3$.

Estimation of $I_{1}$. We immediately have

$$
I_{1} \ll \int_{X-H}^{X}\left(\theta\left((x+H)^{1 / k}\right)-\theta\left(X^{1 / k}\right)-\left((x+H)^{1 / k}-X^{1 / k}\right)\right)^{2} \mathrm{~d} x+H .
$$

By trivial estimates we obtain

$$
I_{1} \ll \log ^{2} X \int_{X-H}^{X}\left((x+H)^{1 / k}-X^{1 / k}\right)^{2} \mathrm{~d} x+H \ll H^{3} X^{2 / k-2} \log ^{2} X+H .
$$


Estimation of $I_{3}$. A similar argument gives the same bound for $I_{3}$, too: we omit it for brevity.

Estimation of $I_{2}$. We have

$$
\begin{aligned}
I_{2} & \ll \int_{X}^{2 X}\left(\theta\left((x+H)^{1 / k}\right)-\theta\left(x^{1 / k}\right)-\left((x+H)^{1 / k}-x^{1 / k}\right)\right)^{2} \mathrm{~d} x+X \\
& =J_{k}(X, H)+X,
\end{aligned}
$$

where we used the definition in (12). Therefore, by (14)-(16), the bound $Y \geq 1 / X$ and recalling that $H=1 /(2 Y)$, we have

$$
\mathcal{I} \ll \frac{X^{2 / k-2} \log ^{2} X}{Y}+X Y^{2}+Y^{2} J_{k}\left(X, \frac{1}{2 Y}\right)
$$

and this proves Theorem 2 .

3.2. Proof of Theorem [3. We reduce our problem to estimate

$$
J_{k, \psi}(X, h):=\int_{X}^{2 X}\left(\psi\left((x+h)^{1 / k}\right)-\psi\left(x^{1 / k}\right)-\left((x+h)^{1 / k}-x^{1 / k}\right)\right)^{2} \mathrm{~d} x
$$

since, using $|a+b|^{2} \leq 2|a|^{2}+2|b|^{2}$ and Lemma 7 below, we have

$$
\begin{aligned}
J_{k}(X, h) & \ll J_{k, \psi}(X, h)+\int_{X}^{2 X}\left(\psi\left((x+h)^{1 / k}\right)-\psi\left(x^{1 / k}\right)-\theta\left((x+h)^{1 / k}\right)+\theta\left(x^{1 / k}\right)\right)^{2} \mathrm{~d} x \\
& \ll J_{k, \psi}(X, h)+h X^{1 / k} .
\end{aligned}
$$

To estimate the right-hand side of (18), we use the following result we will prove later.

Lemma 5. Let $k>0$ be a real number and $\varepsilon$ be an arbitrarily small positive constant. Assuming that (13) holds, there exists a positive constant $c_{1}=c_{1}(\varepsilon)$, which does not depend on $k$, such that

$$
J_{k, \psi}(X, h) \ll h^{2} X^{2 / k-1} \exp \left(-c_{1}\left(\frac{\log X}{\log \log X}\right)^{1 / 3}\right)
$$

uniformly for $X^{1-2 /(C k)+\varepsilon} \leq h \leq X$, where $J_{k, \psi}(X, h)$ is defined in (17). Assuming further that the RH holds, we have

$$
J_{k, \psi}(X, h) \ll h X^{1 / k} \log ^{2}\left(\frac{2 X}{h}\right)
$$

uniformly for $X^{1-1 / k} \leq h \leq X$.

Theorem 3 is an immediate consequence of Lemma 5 and (18). In its turn, Lemma 5 is a consequence of the following result.

Lemma 6. Let $k>0$ be a a real number and $\varepsilon$ be an arbitrarily small positive constant. Assuming that (13) holds, there exists a positive constant $c_{1}=c_{1}(\varepsilon)$, which does not depend on $k$, such that

$$
\begin{aligned}
\widetilde{J}_{k, \psi}(X, \delta) & :=\int_{X}^{2 X}\left(\psi\left((x+\delta x)^{1 / k}\right)-\psi\left(x^{1 / k}\right)-\left((x+\delta x)^{1 / k}-x^{1 / k}\right)\right)^{2} \mathrm{~d} x \\
& \ll \delta^{2} X^{2 / k+1} \exp \left(-c_{1}\left(\frac{\log X}{\log \log X}\right)^{1 / 3}\right)
\end{aligned}
$$

uniformly for $X^{-2 /(C k)+\varepsilon} \leq \delta \leq 1$. Assuming further that the RH holds, we have

$$
\widetilde{J}_{k, \psi}(X, \delta) \ll \delta X^{1 / k+1} \log ^{2}\left(\frac{2}{\delta}\right)
$$


uniformly for $X^{-1 / k} \leq \delta \leq 1$.

The same estimates hold if we insert $\theta$ in place of $\psi$ in the previous quantities.

Proof of Lemma 6. We set $\Delta=(1+\delta)^{1 / k}-1$ in (19) getting

$$
\widetilde{J}_{k, \psi}(X, \delta)=\int_{X}^{2 X}\left(\psi\left(x^{1 / k}(1+\Delta)\right)-\psi\left(x^{1 / k}\right)-\Delta x^{1 / k}\right)^{2} \mathrm{~d} x .
$$

Performing the substitution $y^{k}=x$ we get

$$
\begin{aligned}
\widetilde{J}_{k, \psi}(X, \delta) & =\int_{X^{1 / k}}^{(2 X)^{1 / k}}(\psi(y(1+\Delta))-\psi(y)-\Delta y)^{2} k y^{k-1} \mathrm{~d} y \\
& \asymp_{k} X^{1-1 / k} J\left(X^{1 / k}, \Delta\right) .
\end{aligned}
$$

In the unconditional case, by Lemma 5 of Saffari-Vaughan [11], for $\Delta>X^{-2 /(C k)+\varepsilon}$ we have

$$
\begin{aligned}
\widetilde{J}_{k, \psi}(X, \delta) & \asymp_{k} X^{1-1 / k} \Delta^{2} X^{3 / k} \exp \left(-c_{1}(\varepsilon)\left(\frac{\log X^{1 / k}}{\log \log X^{1 / k}}\right)^{1 / 3}\right) \\
& \asymp_{k} X^{2 / k+1} \Delta^{2} \exp \left(-c_{2}(\varepsilon, k)\left(\frac{\log X}{\log \log X}\right)^{1 / 3}\right),
\end{aligned}
$$

where $c_{1}(\varepsilon)>0$ and we may take, essentially, $c_{2}(\varepsilon, k)=c_{1}(\varepsilon) k^{-1 / 3}$. For $k \in(0,1)$ we are therefore allowed to take a value of $c_{2}$ which is independent of $k$.

In the conditional case, from (20) and by Lemma 5 of Saffari-Vaughan [11, we deduce

$$
\widetilde{J}_{k, \psi}(X, \delta) \asymp_{k} X^{1-1 / k} \Delta X^{2 / k} \log \left(\frac{2 X^{1 / k}}{\Delta}\right) \asymp_{k} X^{1 / k+1} \Delta \log \left(\frac{2 X}{\Delta}\right) .
$$

provided that $\Delta>X^{-1 / k}$. It is easy to see that $\Delta=(1+\delta)^{1 / k}-1=(1 / k) \delta+\mathcal{O}_{k}\left(\delta^{2}\right)$, so that $1 / \Delta=k / \delta+\mathcal{O}_{k}(1) \ll_{k} 1 / \delta$. Hence

$$
\widetilde{J}_{k, \psi}(X, \delta) \asymp_{k} \begin{cases}X^{2 / k+1} \delta^{2} \exp \left(-c_{2}(\varepsilon, k)\left(\frac{\log X}{\log \log X}\right)^{1 / 3}\right) & \text { unconditionally, } \\ X^{1 / k+1} \delta \log \left(\frac{2 X}{\delta}\right) & \text { assuming RH. }\end{cases}
$$

A similar computation allows us to express the above bounds for $\Delta$ in terms of $\delta$. Skipping details for brevity, we may conclude that Lemma 6 holds true for $k \in(0,1)$ (with a constant $c_{1}>0$ that depends only on $\varepsilon$ ) provided that $\delta>X^{-2 /(C k)+2 \varepsilon}$ unconditionally, and that $\delta>(1+\varepsilon) k X^{-1 / k}$ if we assume the $\mathrm{RH}$.

In the remaining range $k>1$ the previous proof gives a constant $c_{1}$ which depends on $k$. In fact, one can obtain Lemma 6 in the full range for $k$ and $c_{1}$ independent from $k$ following the proof of Saffari-Vaughan [11] (as in [7] for the case $k=2$ ) but, since in the applications $k$ is usually bounded, we omit such a proof here.

We finally remark that the estimates with $\theta$ in place of $\psi$ follow arguing as in (18) and using the second part of Lemma 7 ,

Proof of Lemma 5. We follow the argument of $\S 6$ in Saffari-Vaughan [1]. Let now $2 h \leq v \leq 3 h$. and define

$$
\mathfrak{D}_{k, \psi}(a, b)=\psi\left(a^{1 / k}\right)-\psi\left(b^{1 / k}\right)-\left(a^{1 / k}-b^{1 / k}\right) .
$$

To estimate $J_{k, \psi}(X, h)$ (defined in (17)), we first remark, by (21), that

$$
h J_{k, \psi}(X, h) \ll \int_{X}^{2 X} \int_{2 h}^{3 h} \mathfrak{D}_{k, \psi}^{2}(x+v, x) \mathrm{d} v \mathrm{~d} x+\int_{X}^{2 X} \int_{2 h}^{3 h} \mathfrak{D}_{k, \psi}^{2}(x+v, x+h) \mathrm{d} v \mathrm{~d} x .
$$


Setting $z=v-h, y=x+h$ and changing variables in the last integration, the right-hand side of (22) becomes

$$
\ll \int_{X}^{2 X} \int_{2 h}^{3 h} \mathfrak{D}_{k, \psi}^{2}(x+v, x) \mathrm{d} v \mathrm{~d} x+\int_{X+h}^{2 X+h} \int_{h}^{2 h} \mathfrak{D}_{k, \psi}^{2}(y+z, y) \mathrm{d} z \mathrm{~d} y .
$$

Since both the integrand functions are non-negative, we can extend the integration ranges to get

$h J_{k, \psi}(X, h) \ll \int_{X}^{2 X+h} \int_{h}^{3 h} \mathfrak{D}_{k, \psi}^{2}(x+v, x) \mathrm{d} v \mathrm{~d} x=\int_{X}^{2 X+h} x \int_{h / x}^{3 h / x} \mathfrak{D}_{k, \psi}^{2}(x+\delta x, x) \mathrm{d} \delta \mathrm{d} x$,

where in the last step we made the change of variable $\delta=v / x$, thus getting $\delta \geq h / x \geq$ $X^{-2 /(C k)+\varepsilon}$ as in the hypothesis of Lemma 6. Interchanging the integration order we obtain

$$
h J_{k, \psi}(X, h) \ll(X+h) \int_{h /(2 X+h)}^{3 h / X} \int_{X}^{2 X+h} \mathfrak{D}_{k, \psi}^{2}(x+\delta x, x) \mathrm{d} x \mathrm{~d} \delta .
$$

Finally, in the first case, i.e. assuming (13), we use Lemma 6 to get

$$
J_{k, \psi}(X, h) \ll h^{2} X^{2 / k-1} \exp \left(-c_{1}\left(\frac{\log X}{\log \log X}\right)^{1 / 3}\right) .
$$

Assuming RH, Lemma 6 implies

$$
J_{k, \psi}(X, h) \ll \frac{X+h}{h} \int_{h /(2 X+h)}^{3 h / X} \delta X^{1 / k+1} \log ^{2}\left(\frac{2}{\delta}\right) \mathrm{d} \delta \ll h X^{1 / k} \log ^{2}\left(\frac{2 X}{h}\right) .
$$

This concludes the proof of Lemma 5 .

The following elementary lemma is useful in passing from the $\theta$ to the $\psi$ function in Theorem 3 .

Lemma 7. Let $k>0$ be a real number. For $X^{1-1 / k} \leq h \leq X$, we have

$$
\int_{X}^{2 X}\left(\psi\left((x+h)^{1 / k}\right)-\psi\left(x^{1 / k}\right)-\theta\left((x+h)^{1 / k}\right)+\theta\left(x^{1 / k}\right)\right)^{2} \mathrm{~d} x \ll h X^{1 / k} .
$$

Moreover, for $X^{-1 / k} \leq \delta \leq 1$, we have

$$
\int_{X}^{2 X}\left(\psi\left((x+\delta x)^{1 / k}\right)-\psi\left(x^{1 / k}\right)-\theta\left((x+\delta x)^{1 / k}\right)+\theta\left(x^{1 / k}\right)\right)^{2} \mathrm{~d} x \ll \delta X^{1 / k+1} .
$$

Proof. Since $\psi(u)=\sum_{m=1}^{\log _{2} u} \theta\left(u^{1 / m}\right)$, we have

$$
\begin{aligned}
& \psi\left((x+h)^{1 / k}\right)-\psi\left(x^{1 / k}\right)-\theta\left((x+h)^{1 / k}\right)+\theta\left(x^{1 / k}\right) \\
& =\sum_{m=2}^{\log _{2}\left(x^{1 / k}\right)}\left(\theta\left((x+h)^{1 / m k}\right)-\theta\left(x^{1 / m k}\right)\right)+\sum_{m=\log _{2}\left(x^{1 / k}\right)}^{\log _{2}\left((x+h)^{1 / k}\right)} \theta\left((x+h)^{1 / m k}\right) .
\end{aligned}
$$

Clearly, the last sum has at most $1+1 / k$ summands, which are uniformly bounded.

Assume now that $h \in\left[X^{1-1 / k}, X^{1-1 / 2 k}\right]$ and denote as $\Delta_{k}(X, h)$ the left-hand side of the inequality in the statement. Using (23), we find that

$$
\begin{aligned}
\Delta_{k}(X, h) & =\int_{X}^{2 X}\left(\sum_{m=2}^{\log _{2}(x) / k}\left(\theta\left((x+h)^{1 / m k}\right)-\theta\left(x^{1 / m k}\right)\right)+\mathcal{O}(1)\right)^{2} \mathrm{~d} x \\
& \ll \int_{X}^{2 X}\left(\theta\left((x+h)^{1 / 2 k}\right)-\theta\left(x^{1 / 2 k}\right)\right)^{2} \mathrm{~d} x
\end{aligned}
$$




$$
+\int_{X}^{2 X}\left(\sum_{m=3}^{\log _{2}(x) / k}\left(\theta\left((x+h)^{1 / m k}\right)-\theta\left(x^{1 / m k}\right)\right)\right)^{2} \mathrm{~d} x+\mathcal{O}(X) .
$$

We only deal with the first term, the other one being similar and, in fact, smaller. We exploit the fact that the integrand is usually 0 and small when positive. Let $M=\left\lceil X^{1 / 2 k}\right\rceil$ and $N=\left\lfloor(2 X)^{1 / 2 k}\right\rfloor$. Let $n \in[M, N]$; if $x \in[X, 2 X]$ satisfies both $x^{1 / 2 k}<n$ and $(x+h)^{1 / 2 k} \geq n$, then the integrand $\left(\theta\left((x+h)^{1 / 2 k}\right)-\theta\left(x^{1 / 2 k}\right)\right)^{2}$ is $\log ^{2} n$ if $n$ is a prime number, and vanishes otherwise. The two inequalities above imply $n^{2 k}-h \leq x<n^{2 k}$. Summing up, for every prime $p \in[M, N]$ there is an interval of length $h$ of values for $x$ such that the integrand does not vanish. Hence

$$
\Delta_{k}(X, h) \ll h \sum_{p \in[M, N]}(\log p)^{2} \ll h X^{1 / 2 k} \log X .
$$

Let us consider now $h \in\left[X^{1-1 / 2 k}, X\right]$. For the terms with $m \geq 3$ in (23) we simply notice that

$$
\begin{aligned}
\theta\left((x+h)^{1 / m k}\right)-\theta\left(x^{1 / m k}\right) & \leq \log \left((x+h)^{1 / m k}\right) \sum_{x^{1 / m k}<n \leq(x+h)^{1 / m k}} 1 \\
& \leq \frac{1}{m k} \log (x+h)\left((x+h)^{1 / m k}-x^{1 / m k}+1\right) \\
& \leq \frac{1}{m k} \log (x+h)\left(\frac{1}{m k} h x^{1 / m k-1}+1\right)
\end{aligned}
$$

by the mean-value theorem. The number of such terms is at most $\log x$ and hence the total contribution is bounded by an absolute constant times $\log x \log \log x\left(h x^{1 / 3 k-1}+1\right)$. We now deal with the term $\theta\left((x+h)^{1 / 2 k}\right)-\theta\left(x^{1 / 2 k}\right)$. We have

$$
\begin{aligned}
\theta\left((x+h)^{1 / 2 k}\right)-\theta\left(x^{1 / 2 k}\right) & \leq \log \left((x+h)^{1 / 2 k}\right)\left(\pi\left((x+h)^{1 / 2 k}\right)-\pi\left(x^{1 / 2 k}\right)\right) \\
& \ll_{k} \log x \frac{h x^{1 / 2 k-1}+1}{\log \left(h x^{1 / 2 k-1}+1\right)}
\end{aligned}
$$

by the mean-value theorem again and the Brun-Titchmarsh inequality, which we can use for $h \gg_{k} X^{1-1 / 2 k}$. Squaring out and integrating the previous estimates we get, for every fixed $\varepsilon>0$, that

$$
\Delta_{k}(X, h) \ll_{k, \varepsilon} \begin{cases}h^{2} X^{1 / k-1} & \text { if } X^{1-1 /(2 k)+\varepsilon} \leq h \leq X, \\ h^{2} X^{1 / k-1} \log ^{2} X & \text { if } X^{1-1 /(2 k)} \leq h \leq X^{1-1 /(2 k)+\varepsilon} .\end{cases}
$$

The first part of Lemma 7 now follows from (24)-(25) by trivial computations.

The second part of Lemma 7 can be obtained with a similar argument.

\section{REFERENCES}

[1] R. C. Baker and G. Harman, Diophantine approximation by prime numbers, J. London Math. Soc. 25 (1982), 201-215.

[2] J. Brüdern, R. J. Cook, and A. Perelli, The values of binary linear forms at prime arguments, Proc. of Sieve Methods, Exponential sums and their Application in Number Theory (G. R. H. Greaves et al, ed.), Cambridge U.P., 1997, pp. 87-100.

[3] P. X. Gallagher, A large sieve density estimate near $\sigma=1$, Invent. Math. 11 (1970), 329-339. 
[4] A. Ghosh, The distribution of $\alpha p^{2}$ modulo 1, Proc. London Math. Soc. 42 (1981), no. 2, 252-269.

[5] G. Harman, Diophantine approximation by prime numbers, J. London Math. Soc. 44 (1991), 218-226.

[6] M. N. Huxley, On the difference between consecutive primes, Invent. Math. 15 (1972), $155-164$.

[7] A. Languasco and V. Settimi, On a Diophantine problem with one prime, two squares of primes and s powers of two, Acta Arithmetica 154 (2012), 385-412.

[8] A. Languasco and A. Zaccagnini, A Diophantine problem with a prime and three squares of primes, to appear in J. Number Theory, http://arxiv.org/pdf/1206.0246, 2012.

[9] A. Languasco and A. Zaccagnini, A Diophantine problem with prime variables, http://arxiv.org/pdf/1206.0252, Submitted, 2012.

[10] G. J. Rieger, Uber die Summe aus einem Quadrat und einem Primzahlquadrat, J. Reine Angew. Math. 231 (1968), 89-100.

[11] B. Saffari and R. C. Vaughan, On the fractional parts of $x / n$ and related sequences. II, Ann. Inst. Fourier 27 (1977), 1-30.

[12] D. Tolev, On a Diophantine inequality involving prime numbers, Acta Arith. 61 (1992), 289-306.

[13] R. C. Vaughan, Diophantine approximation by prime numbers. I, Proc. London Math. Soc. 28 (1974), 373-384.

[14] R. C. Vaughan, Diophantine approximation by prime numbers. II, Proc. London Math. Soc. 28 (1974), 385-401.

[15] R. C. Vaughan, The Hardy-Littlewood method, second ed., Cambridge U. P., 1997.

[16] A. Zaccagnini, Primes in almost all short intervals, Acta Arith. 84.3 (1998), 225244.

Alessandro LANGUASCO

Università di Padova

Dipartimento di Matematica

Via Trieste 63

35121 Padova, Italy

E-mail: languasco@math.unipd.it

Alessandro ZACCAGNINI

Università di Parma

Dipartimento di Matematica

Parco Area delle Scienze, 53/a

43124 Parma, Italy

E-mail: alessandro.zaccagnini@unipr.it 